\title{
Cisj
}

\section{NEURAL-BASED RECOGNITION OF MULTI-PARAMETER SENSOR SIGNAL DESCRIBED BY MATHEMATICAL MODEL}

\author{
Iryna Turchenko', Volodymyr Kochan², Anatoly Sachenko ${ }^{3}$ \\ Institute of Computer Information Technologies \\ Ternopil Academy of National Economy \\ 3 Peremoga Square, Ternopil 46004 Ukraine \\ 1)vtu@tanet.edu.te.ua, 2)vk@tanet.edu.te.ua, 3) as@tanet.edu.te.ua
}

\begin{abstract}
The possibility of artificial neural network usage for recognition of a signal of a multi-parameter sensor is described in this paper. The general structure of data acquisition channel with usage of neural networks as well as mathematical model of output signal of a multi-parameter sensor is studied in this article. The model of neural network, training algorithm and achieved results of simulation modeling of a multi-parameter sensor signal recognition using MATLAB software are presented at the end of this paper.
\end{abstract}

Keywords: a multi-parameter sensor, sensor signal recognition, neural networks.

\section{INTRODUCTION}

Sensitivity to one measuring physical quantity was always one of the main requirements to sensors. The dependence of a sensor output signal on other physical quantities was considered to be its disadvantage. Such physical quantities are called influence quantities. Their influence on data acquisition results is compensated by: (i) development of improved sensors [1] for which the influence of other physical quantities does not exceed an admissible error (constructive and technological methods of sensor improvement); (ii) appropriate correction [2] of output sensor signal (structure and algorithmic methods). For thermocouples such improvement includes: (i) development of the B-type thermocouple with influence error which does not exceed 0.3 of the admissible error; (ii) correction of free point temperature influence by hardware way (bridgecircuit using thermometer of resistance) or by computing way (subroutine using data acquisition results of another measurement channel).

However, the sensors with output signal specially depended on several physical quantities are widely used recently. These sensors are called multiparameter sensors (MPS). Such sensors are used for simultaneous data acquisition of several physical quantities [3]. If the different parameters of MPS output signal depend on separate physical quantities, a problem with construction of appropriate data acquisition system does not arise. However such situation is not typical for MPS. Measuring physical quantities more often influence on all the parameters of MPS output signal. For example, MPS sensitive element is very often implemented as a film, which contacts with measured physical quantities. In particularly, such sensors are used for monitoring of different types of pollution during ecological researches. Similar sensors are also used for identification of explosive gases [4] in mineral resource industry. These sensors are constructed on the basis of $\mathrm{SnO}_{2}$ film, which senses the density of $\mathrm{CO}$ and $\mathrm{CH}_{4}$ gases while the film is heating by electric current. The conductivity, which depends on both physical quantities, is normally the output value of MPS. Therefore there is an urgent problem to recognize (identify) the information from MPS output signal in order to calculate the results of data acquisition of separate physical quantities.

Normally the systems with MPS use dependences of output signal sensitivity on measured physical quantities in different operation modes for calculation of data acquisition results of different physical quantities. Thus the following ways are used for this purpose: 
1. to find such MPS operation modes at which the sensitivity to each measured physical quantity dominates considerably over the sensitivity to other physical quantities. Further it is necessary to reconstruct founded MPS operation modes and evaluate output signals. If the influence of other physical quantities does not exceed the admissible error then this error is neglected. In such a case the processing of MPS output signal does not differ from processing of traditional sensor output signal. It is a considerable advantage of this method. The defect of this method is absence of universality. It can be used only for some types of MPS for which it is possible to find and implement the appropriate operation modes in real exploitation conditions;

2. to find such MPS operation modes at which the sensitivity to each measured physical quantity predominates over the sensitivity to other physical quantities. Further it is necessary to describe the set of equations. The solution of the equations allows defining the values of separate physical quantities. The defect of this method is the necessity of additional researches for definition: (i) the coefficients of the equations' set, solution of which allows defining measured physical quantities; (ii) the link between three values such as: (1) the errors of calculation of the coefficients, (2) the errors of MPS output signal measurement in all allowable operation modes, and (3) the errors of data calculation of measuring physical quantities.

The similar task is arising during fonts or symbols recognition where artificial neural network applications provide successful results.

Neural networks are widely used by other research teams for recognition of MPS signals. In paper [5] the analysis of seven well-known methods of MPS signals pattern recognition based on classical mathematical approaches as well as on neural networks is fulfilled. In particularly, there are methods of (i) nearest neighbor, (ii) Mahalanobis linear discriminant analysis, (iii) Bayes discriminant analysis, (iv) soft independent modeling of class analogy, (v) artificial neural network based on back-propagation training, (vi) probabilistic neural networks and (vii) leaning vector quantization. This analysis shows that methods of artificial neural network, probabilistic neural networks and leaning vector quantization, i.e. the methods based on neural network approach, are more accurate.

Also there are different kinds of neural network used, in particularly neural networks with radial- basic activation function [6], time delay neural networks [7], neural networks based on adaptive resonance theory [8], self-organizing maps [9] and multi-layer perceptron with back-propagation error training [10-13]. All these approaches lead to wide application areas. For example, analysis of gas concentrations in mixtures in industrial applications and environment is considered in [8-11, 14-15], analysis of odors and fragrant materials $[6,7,10]$, chemical compounds in dielectric ceramics in electronics [13].

This analysis also has shown that the majority of the implementations described above use large number of sensor data gathered from sensors arrays. Only one paper [9] deals with one MPS gathering data by the change of its operation mode similar [4] described above. Therefore it is proposed below to consider the approach of artificial neural network usage for identification of separate physical quantities from single MPS signal in multi-sensor data acquisition system.

\section{GENERAL STRUCTURE OF NEURAL- EMBEDDED DATA ACQUISITION CHANNEL OF MPS}

The general structure of MPS data acquisition channel is presented in Fig. 1. The physical quantities F1 and F2 go to MPS input. The output MPS signal SS goes to analog circuit AC (amplifier). Further the amplified signal goes to analog-to-digital converter ADC. The output ADC code $\mathrm{N}$ goes to the microcontroller MC. The MC using signal "MS Mode" controls MPS operating modes. The signal AS corresponds to operating modes with different sensitivity to physical quantities F1 and F2. As a result of AS signal conversion into code the MC gives the codes NM1 and NM2 to neural network NN. These codes are used for calculation of the values of input physical quantities $\mathrm{F} 1 *$ and $\mathrm{F} 2 *$.

The advantage of such structure is absence of necessity to analyze deeply the results of MPS experimental researches for identification of both dependences $\mathrm{F}^{*}$ and $\mathrm{F} 2 *$ from $\mathrm{F} 1$ and $\mathrm{F} 2$ with given accuracy. The neural network can identify the nature of sensor signal due to its self-training capabilities. It is necessary to provide only highquality $\mathrm{NN}$ training for this purpose using the training set based on sufficient number of experimental data. However it is necessary to note, that the computing resources needed for $\mathrm{NN}$ training $10^{3} \ldots 10^{4}$ times exceed the computing resources needed for usage of trained NN [16]. Therefore it is expedient to train $\mathrm{NN}$ on separate 
personal computer with appropriate computing power and to include it to the hardware structure of the data acquisition system based on MPS. A general structure of $\mathrm{NN}$ training in MPS data acquisition channel is presented in Fig. 2.

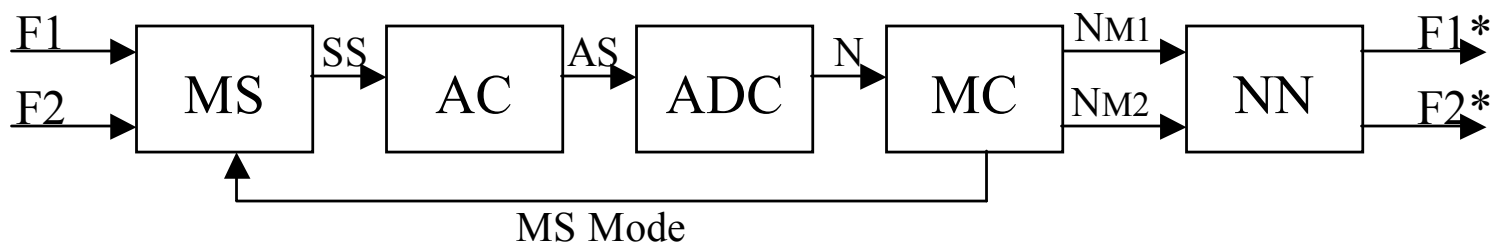

Fig. 1 - A general Structure of Neural-embedded Data Acquisition Channel of MPS

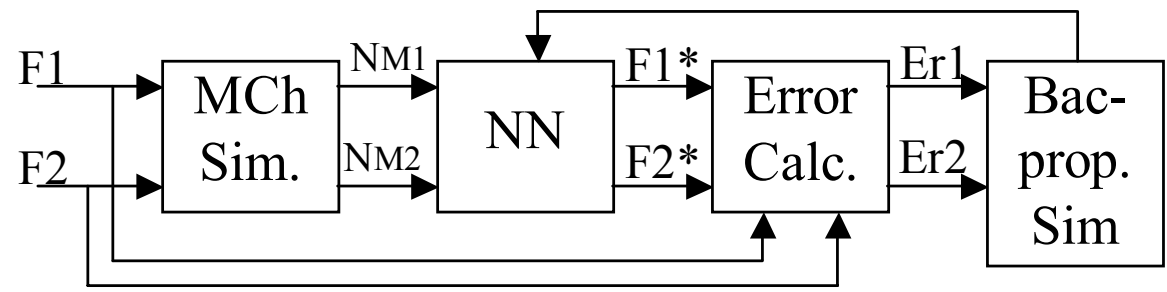

Fig. 2 - A general structure of NN training in MPS data acquisition channel

It is necessary to put the output codes NM1 and NM2 of the microcontroller MC (see Fig. 1) to NN inputs in order to train it using the results of MPS experimental researches. It is necessary to use simulator of the data acquisition channel MCh Sim (see Fig. 2) for this purpose. Then the values of input physical quantities $F 1$ and F2, which correspond to physical quantities during experimental researches, go to MCh Sim input. On $\mathrm{NN}$ output we will receive the calculation results of physical quantities $\mathrm{F} 1 *$ and $\mathrm{F} 2 *$. These results are compared with values F1 and F2. As the final result we obtain the values of Er1 and Er2 errors, which go to "Bacprop" unit for changing of $\mathrm{NN}$ weight coefficients and thresholds. It is necessary to choose the expedient and preferred NN structures and their training algorithms experimentally. Also it is expedient to research dependence between calculation error of separate physical quantities and $\mathrm{NN}$ training parameters for implementation of real data acquisition systems based on MPS. Thus such research is expedient to carry out on mathematical model of a multi-parameter sensor.

\section{MATHEMATICAL MODEL OF MPS OUTPUT SIGNAL}

The analysis of the types of conversion characteristics of existing MPS [4] allowed selecting their main features:

- they can be described by functions of two variables;

- these characteristics have nonlinear character;

- there is a considerable correlation link between the characteristics in different operating modes.
According to these features, the mathematical model of MPS output signal could be presented as multiplication of two different-order polynomials with different coefficients:

$$
Y_{1}=\left(A_{1} \times X_{1}^{k}+B_{1} \times X_{1}\right) \times\left(C_{1} \times X_{2}^{l}+D_{1} \times X_{2}\right)
$$

As it is described in Section 2, it is necessary to receive MPS output signal in several operating modes with different sensitivity to different physical quantities. The different operating modes of MPS are modeled choosing the different values of the polynomials orders $k$ and $l$ and A, B, C and D coefficients.

The simulation models, which cover all the types of combinations of MPS conversion characteristics on different physical quantities, were designed:

1. The MPS conversion characteristics have positive derivatives on both physical quantities;

2. The MPS conversion characteristics have negative derivatives on both physical quantities;

3. The MPS conversion characteristics have derivatives with different sign on both physical quantities.

The view of first simulation model is presented in Fig. 3 and Fig. 4. The graphics of MPS output signal dependence from two physical quantities according to the first combination of the conversion characteristics is presented in Fig. 3. This dependence is a surface shown in Fig. 3 as the graphs of sectional views. Thus the values of one physical quantity are shown on abscissa axis, and each curve corresponds to one value of other physical quantity. The values of physical quantities and MPS output signal are presented in conventional units in order to provide simulation 
modeling. The similar dependence of output signal of this MPS in another operation mode is presented in Fig. 4.

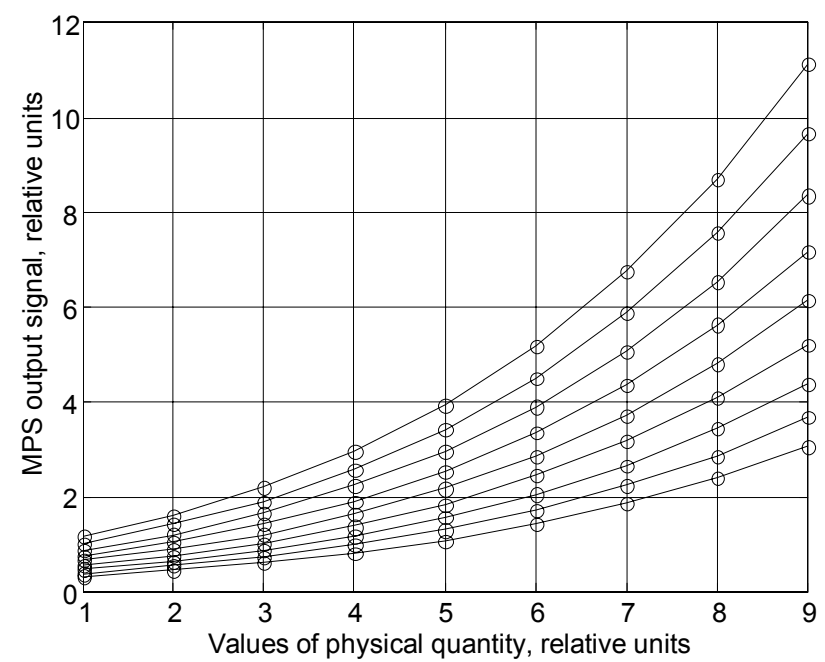

Fig. 3 - Dependence of output signal of MPS in the first operation mode

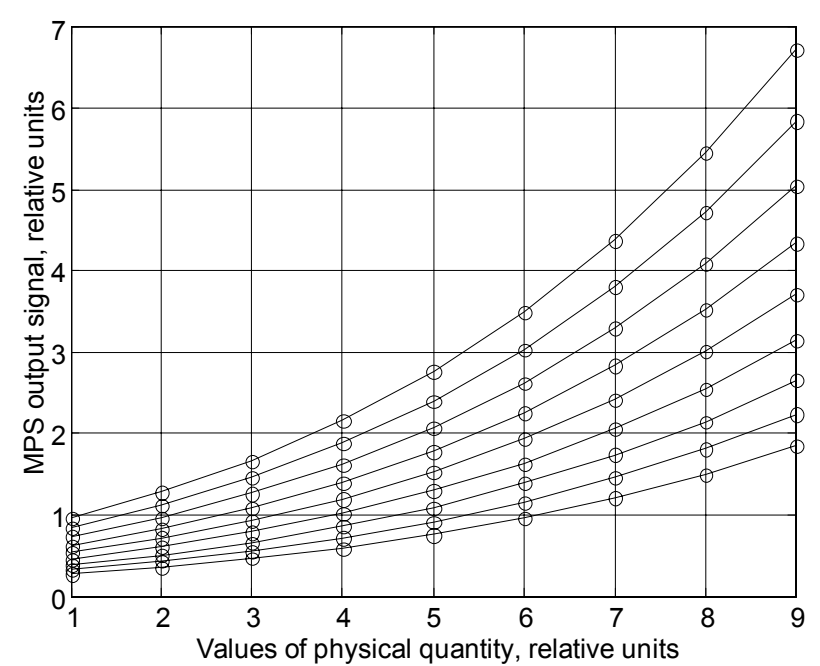

Fig. 4 - Dependence of output signal of MPS in the second operation mode

\section{STRUCTURE OF NEURAL NETWORK FOR MPS SIGNAL IDENTIFICATION}

It is obvious, that the number of neurons of NN output layer should correspond to the number of the input physical quantities of MPS. The number of neurons of $\mathrm{NN}$ input layer is defined by the number of operation modes implemented in the structure of Fig. 1. Two input and two output neurons are the minimum needed neurons for MPS used for data acquisition of two physical quantities. From the one hand, the number of neurons of the hidden layer should provide sufficient generalized properties of neural network. However from the other hand the increasing of the number of neurons in the hidden layer can make generalized properties of neural network worse and convert such neural network into associative memory. Among known NN structures solving recognition and identification tasks, it is expedient to select multi-layer perceptron structure because it is sufficient for preliminary experimental researches [17]. Therefore the multilayer perceptron with 2 inputs, $\mathrm{N}$ hidden and 2 output neurons is chosen for identification of the values of separate physical quantities of MPS signal (Fig. 5).

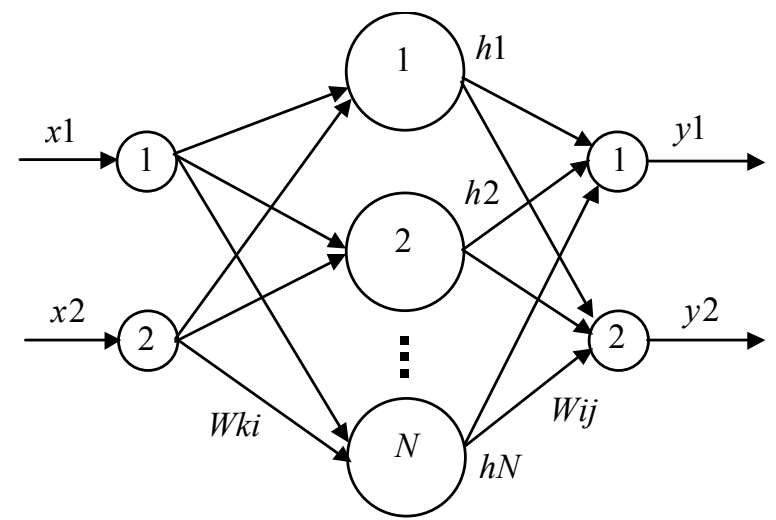

Fig. 5 - Neural network identification of the values of separate physical quantities of MPS signal

The output of any neuron of previous layer is connected to inputs of all next-layer's neurons by synapses. Therefore this perceptron has regular structure. Output value the $j$-neuron of the output layer is

$$
y_{j}=F_{3}\left(\sum_{i=1}^{N} w_{i j} h_{i}-T_{j}\right) \text {, }
$$

where $F_{3}(x)$ is the activation function of the neurons of the output layers, $w_{i j}$ is the synapse from $i$-neuron of the hidden layer to $j$-neuron of the output layer, $h_{i}$ is the output value of $i$-neuron of the hidden layer, $T_{j}$ is the threshold of $j$-neuron of the output layer.

The output value of $i$-neuron of the hidden layer is

$$
h_{i}=F_{2}\left(\sum_{k=1}^{2} w_{k i} x_{k}-T_{i}\right) \text {, }
$$

where $F_{2}(x)$ is the activation function of the neurons of the hidden layer, $w_{k i}$ is the synapse from $k$-input neuron to $i$-neuron of the hidden layer, $x_{k}$ 
is the input value of neural network and $T_{i}$ is the threshold of the $i$-neuron of the hidden layer.

The preliminary analysis of known algorithms of $\mathrm{NN}$ training showed, that it is expedient to use Levenberg-Marquardt algorithm [18]. For example, neural network with usage of Levenberg-Marquardt method is trained 5 times faster and relative identification accuracy is 4 times less. The nonlinear logistic activation function is used for the hidden and output neurons

$$
F(x)=1 /\left(1+e^{-x}\right) .
$$

The Levenberg-Marquardt algorithm was designed to approach second-order training speed without having to compute the Hessian matrix [18]. This matrix approximated with use of the Jacobian matrix which can be computed through a standard backpropagation technique that is much less complex than computing the Hessian matrix. The performance function will always be reduced on the each iteration of the algorithm.

\section{RESULTS OF SIMULATION MODELING}

The software routine of simulation modeling is developed in the MATLAB ${ }^{\circledR}$ version 6.1. There used 81 vectors for NN training. Each of the vectors consists of two input values, which simulate MPS output signal and two output values, which simulate the values of input physical quantities. These 81 vectors were obtained by substitution of any of nine arguments shown in Fig. 3 and Fig. 4 using expression (1). Thus, the training vector appears as the values $X_{1} ; X_{2} ; Y_{1} ; Y_{2}$, where $\left\{X_{1} ; Y_{1} ;\right\}$ are the values describing input-output conversion of the first MPS parameter; $\left\{X_{2} ; Y_{2} ;\right\}$ are the values describing input - output conversion of the second MPS parameter (Fig. 6). The testing set for identification consists of 576 values. It is formed using three points between the integer values from the training set (Fig. 7).

There used the multi-layer perceptron with 2 input, 10 hidden and 2 output neurons. The mathematical model of multi-layer perceptron is described by expressions (2-4). The LevenbergMarquardt algorithm is used for training. A maximum quantity of training epoch is 40000 . As it is seen from Fig. 8, the maximum relative error of $\mathrm{NN}$ training both for the first and second outputs does not exceed $1 \%$ in case of training to sumsquared error $10^{-5}$. A set of simulation modeling researches is carried out for estimation of potential capabilities of the proposed approach.

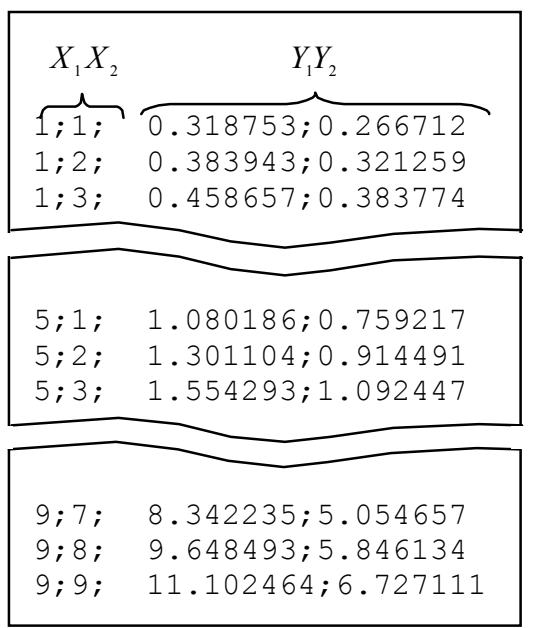

Fig. 6 - Fragment of the training set of neural network

\begin{tabular}{|c|c|}
\hline$\underbrace{X_{1} X_{2}}$ & $\underbrace{}_{Y_{1} Y_{2}}$ \\
\hline $1.25 ; 1.25$ & $0.363069 ; 0.300219$ \\
\hline $1.25 ; 1.50 ;$ & $0.380459 ; 0.314598$ \\
\hline $1.25 ; 1.75 ;$ & $0.398466 ; 0.329488$ \\
\hline$\ldots 25 ; 2.25 ;$ & $0.436389 ; 0.36084$ \\
\hline $1.25 ; 2.50 ;$ & $0.456335 ; 0.377340$ \\
\hline $1.25 ; 2.75 ;$ & $0.476957 ; 0.394392$ \\
\hline $1.25 ; 3.25 ;$ & $0.520290 ; 0.430224$ \\
\hline $1.25 ; 3.50 ;$ & $0.543032 ; 0.449028$ \\
\hline $1.25 ; 3.75 ;$ & $0.566511 ; 0.468443$ \\
\hline $8.75 ; 7.25 ;$ & $8.146524 ; 4.978996$ \\
\hline $8.75 ; 7.50 ;$ & $8.449619 ; 5.164241$ \\
\hline $8.75 ; 7.75 ;$ & $8.761093 ; 5.354608$ \\
\hline $8.75 ; 8.25 ;$ & $9.409795 ; 5.751082$ \\
\hline $8.75 ; 8.50 ;$ & $9.747332 ; 5.957378$ \\
\hline $8.75 ; 8.75 ;$ & $10.093871 ; 6.169176$ \\
\hline
\end{tabular}

Fig. 7 - Fragment of the testing set of neural network

The sum-squared error of $\mathrm{NN}$ training was being varied from $10^{-2}$ up to $10^{-6}$. The following Fig. 9 displays dependence of the maximum relative error of physical quantity identification on the value of sum-squared error. The maximum relative error of identification does not exceed $1 \%$ in case of $\mathrm{NN}$ training to sum-squared errors $10^{-4}, 10^{-5}$ and $10^{-6}$. Thus the average value of relative error of identification does not exceed $0.1 \%$.

The investigation of the dependence of accuracy of physical quantity identification from neural network structure is also considered in this section. In accordance with the task considered above we cannot change the number of input and output neurons, because these neurons are responsible for organizing the information processing by neural 
network, i.e. for receiving the input data and providing the results of identification outside. Therefore we can change only the number of neurons of the hidden layer, those actually provide information processing. This research is necessary to make by training the neural network to sumsquared error up to $10^{-5}$ because in this case neural network provides good identification results (see Fig. 9).

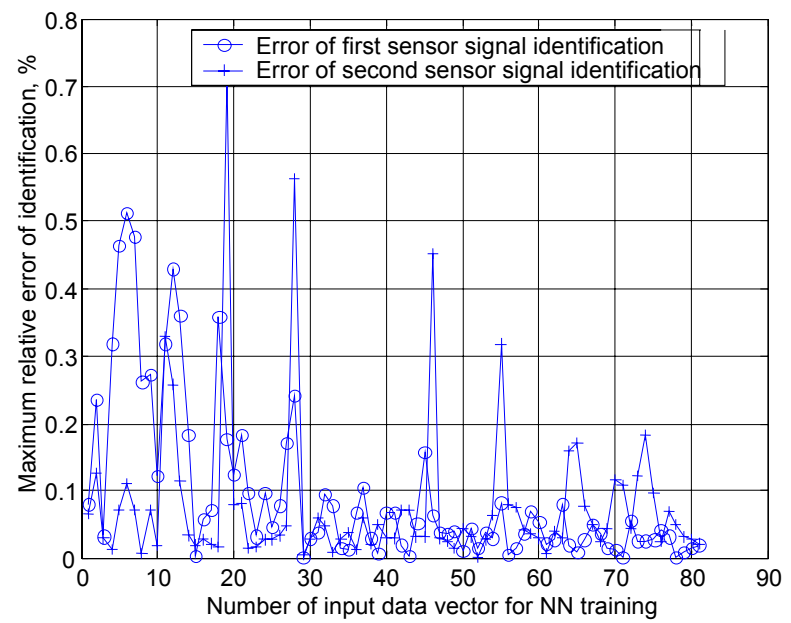

Fig. 8 - Identification of MPS signals inside the training set

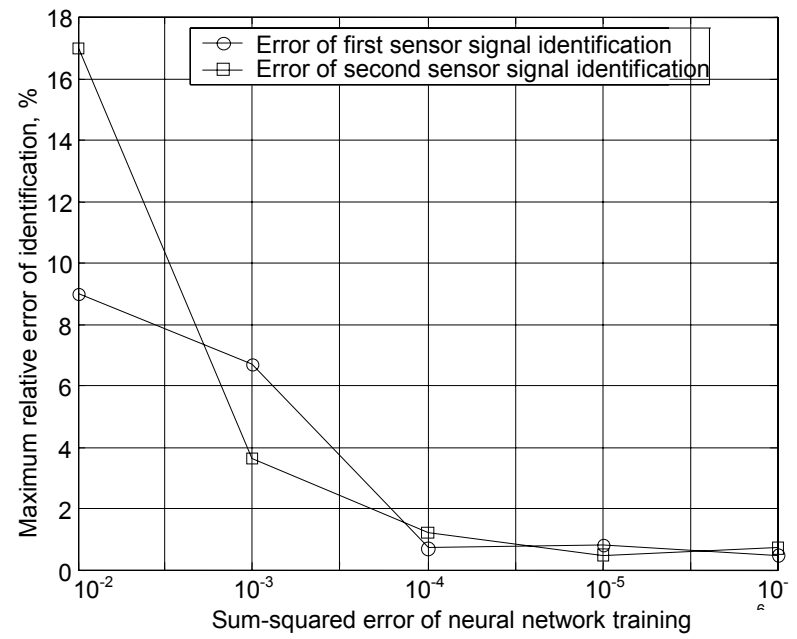

Fig. 9 - Identification of MPS signals outside the training set for $\mathrm{NN}$ with 10 hidden neurons

The dependencies of average and maximum relative identification errors from the number of hidden neurons of neural network are presented in Fig. 10 and Fig. 11 respectively. As it is seen in Fig. 10 , the average relative error of identification does not exceed $3 \%$ at changing the number of hidden layer neurons from 10 till 80 . This shows that potentially artificial neural networks approach is suitable for recognition and identification of the signals of multi-parameter sensors. However, as it is seen in Fig. 11, the maximum relative error of identification considerably grows (up to $120 \%$ ) at increasing the number of neurons of the hidden layer. The analysis of numerical data showed, that maximum error grows exceptionally only in a few points of recognition results and low value of the average error confirms this fact. It can be explained by presence of random component of the signal in the mathematical model of multi-parameter sensor (1). The increasing of maximum relative error of identification in this case confirms the known thesis of artificial neural networks theory. In particularly, if the number of neurons of the hidden layer approaches to the number of input training vectors, this neural network becomes an associative memory and generalized properties of such neural network are considerably get worse.

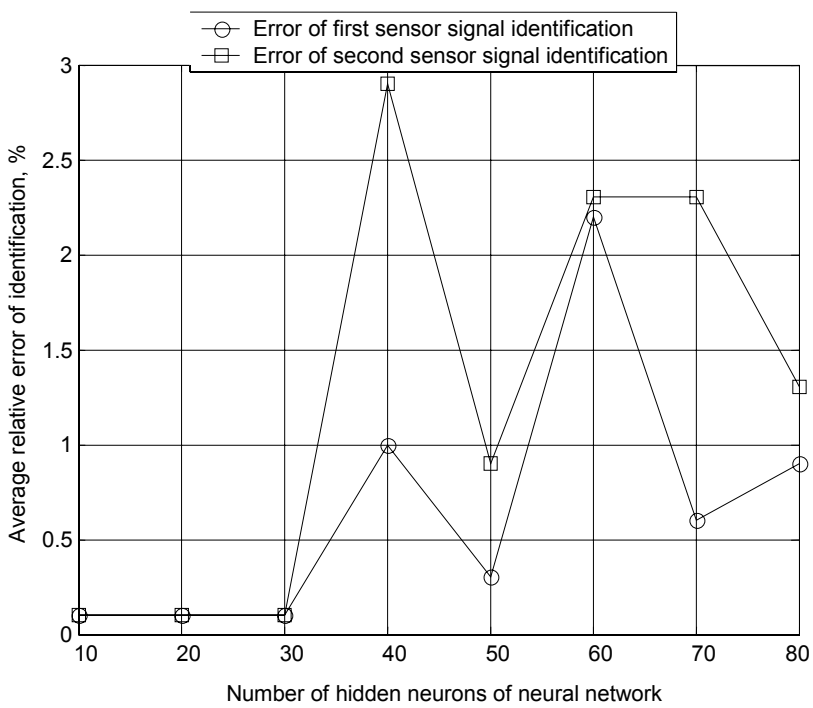

Fig.10 - Dependence of average identification error number of hidden neurons of neural network

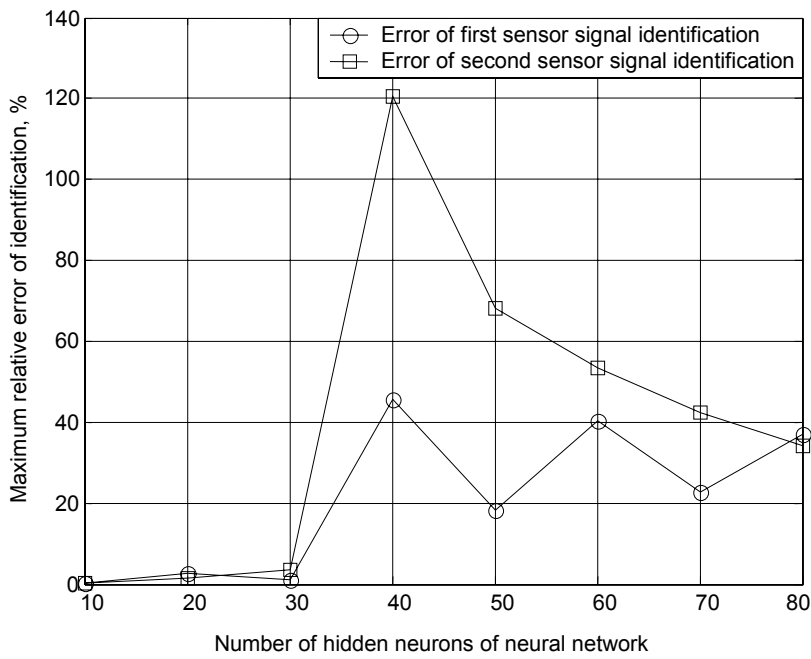

Fig. 11 - Dependence of maximum identification error from number of hidden neurons of neural network

Also this fact is confirmed by the results of training process analysis. For example, during increasing the number of neurons of the hidden layer the relative training error of neural network 
practically remains small and does not exceed $1.6 \%$ (Fig. 12) and the number of training epoch is considerably decreasing (Fig. 13). It can be explained that neural network trains easier at a larger number of the hidden neurons because it is easier to bring into accordance 81 input vectors with 80 hidden neurons than 10 hidden neurons in our case.

Therefore the structure of neural networks 2-10-2 investigated in Fig. 9 is better from accuracy point of view to solve the task of multi-parameter sensor signal recognition using 81 input training vectors.

\section{CONCLUSIONS AND FUTURE RESEARCHES}

The reached values of maximum relative error showed potential capabilities of neural networks to recognize the values of separate physical quantities of a multi-parameter sensor. The simulation modeling results of one combination of conversion characteristics of a multi-parameter sensor for different physical quantities are considered in this paper. It is expedient to carry out future researches using real output signal of a multi-parameter sensor.

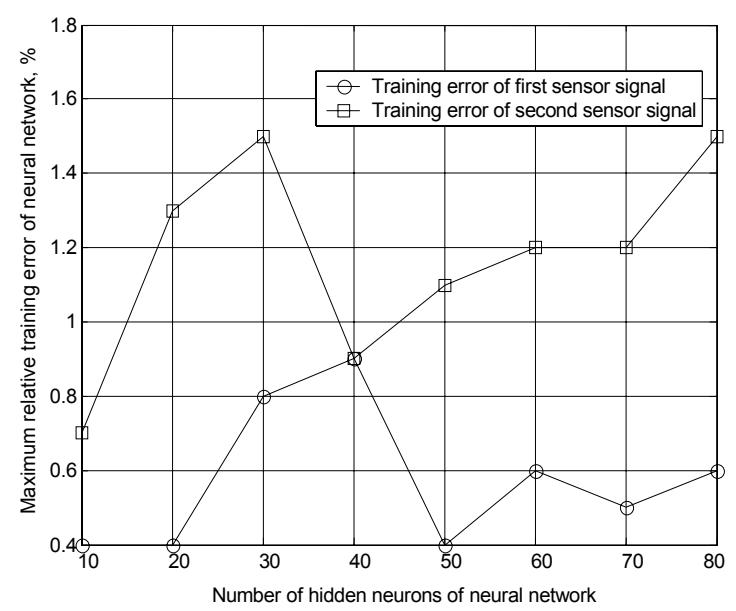

Fig.12 - Dependence of maximum training error from number of hidden neurons of neural network

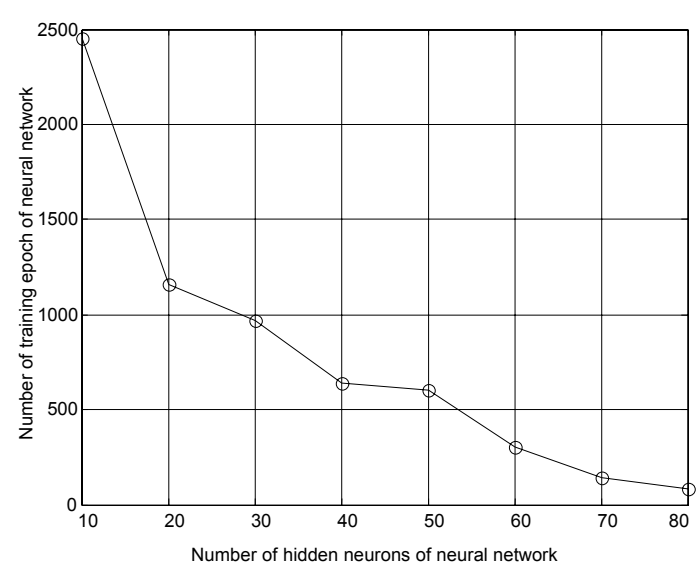

Fig. 13 - Dependence of epoch number from number of hidden neurons of neural network

\section{REFERENCES}

[1] G. Samsonov, A. Kits, O. Kuzdeni, Sensors for temperature measurement in industry, Kiev: Naukova dumka, 1977, 223 p.

[2] Tuz Yu, Structure methods of instrumentation accuracy improvement, Kiev: Vyshcha shkola, 1976, $255 \mathrm{p}$.

[3] http://www.bibl.liu.se/liupubl/disp/disp99/t ek573s.htm

[4] J. Zakrzewski, W. Domanski, P. Chaitas and Th. Laopoulos, "Improving Sensitivity and Selectivity of $\mathrm{SnO} 2$ Gas Sensors by Temperature Variation", Proceedings of the second IEEE International Workshop on Intelligent Data Acquisition and Advanced Computing Systems: Technology and Applications, 2003, Lviv, Ukraine, pp. 296-299.

[5] Shaffer R. E., Rose-Pehrsson S. L., McGill A.R. A comparison study of chemical sensor array pattern recognition algorithms // Analytica Chimica Acta. - 1999. - Vol. 384, No. 3. - P. 305-317.

[6] Daqi G., Shuyan W., Yan J. An electronic nose and modular radial basis function network classifiers for recognizing multiple fragrant materials // Sensors and Actuators B. - 2004. - Vol. 97, No. 2-3. - P. 391-401.

[7] Zhang H., Balaban M., Principe J. Improving pattern recognition of electronic nose data with time-delay neural networks // Sensors and Actuators B. - 2003. - Vol. 96, No. 1-2. - P. 385389.

[8] Llobet E., Brezmes J., Gualdrón O., Vilanova X., Correig X. Building parsimonious fuzzy ARTMAP models by variable selection with a cascaded genetic algorithm: application to multisensor systems for gas analysis // Sensors and Actuators B. - 2004. - Vol. 99, No. 2-3. - P. 267272.

[9] Ortega A., Marco S., Perera A., Šundic T., Pardo A., Samitier J. An intelligent detector based on temperature modulation of a gas sensor with a digital signal processor // Sensors and Actuators B. - 2001. - Vol. 78, No. 1-3. - P. 32-39.

[10] Luo D., Hosseini G., Stewart J. Application of ANN with extracted parameters from an electronic nose in cigarette brand identification // Sensors and Actuators B. - 2004. - Vol. 99, No. 23. - P. 253-257.

[11] Martín M., Santos J., Agapito J. Application of artificial neural networks to calculate the partial gas concentrations in a mixture // Sensors and Actuators B. - 2001. - Vol. 77, No. 12. - P. 468-471.

[12] Srivastava A.K. Detection of volatile organic compounds (VOCs) using $\mathrm{SnO} 2$ gas-sensor 
array and artificial neural network // Sensors and Actuators B. - 2003. - Vol. 96, No. 1-2. - P. 24-37.

[13] Guo D., Wang Y., Nan C., Li L., Xia J. Application of artificial neural network technique to the formulation design of dielectric ceramics // Sensors and Actuators A. - 2002. - Vol. 102, No. 1-2. - P. 93-98.

[14] Capone S., Siciliano P., Bârsan N., Weimar U., Vasanelli L. Analysis of $\mathrm{CO}$ and $\mathrm{CH} 4$ gas mixtures by using a micromachined sensor array // Sensors and Actuators B: Chemical. - 2001. - Vol. 78, No. 1-3. - P. 40-48.

[15] Hahn S., Bârsan N., Weimar U. Investigation of $\mathrm{CO} / \mathrm{CH} 4$ mixture measured with differently doped $\mathrm{SnO} 2$ sensors // Sensors and Actuators B. - 2001. - Vol. 78, No. 1-3. - P. 64-68.

[16] V. Turchenko, V. Kochan, A. Sachenko, "Estimation of Computational Complexity of Sensor Accuracy Improvement Algorithm Based on Neural Networks", Lecture Notes in Computing Science, LNCS 2130, Ed. G. Dorffner, H. Bischof, and K. Hornik, Springer-Verlag, Berlin, Heidelberg, New York, 2001, pp. 743-748.

[17] A. K. Jain, J. Mao, K. M. Mohiuddin, "Artificial neural networks: a tutorial", Computer, 1996, pp. 31-44.

[18] M. T. Hagan and M. Menhaj, "Training feed-forward networks with the Marquardt algorithm", IEEE Transactions on Neural Networks, vol. 5, no. 6, pp. 989-993, 1994

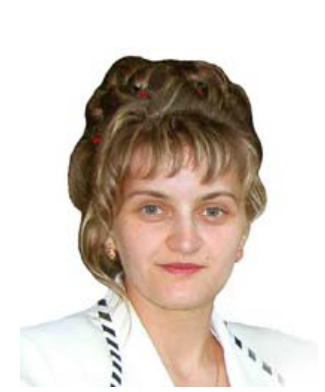

Mrs. Iryna V. Turchenko received her M.S. degree from Ternopil Academy of National Economy, Ukraine in 1997 in management information systems. Now she is pursuing her Ph.D. course in Ternopil Academy of National Economy in field of intelligent computer systems.

Her main research interests are: theory and application of neural networks, multiparameter sensors, intelligent and web-based instrumentations.

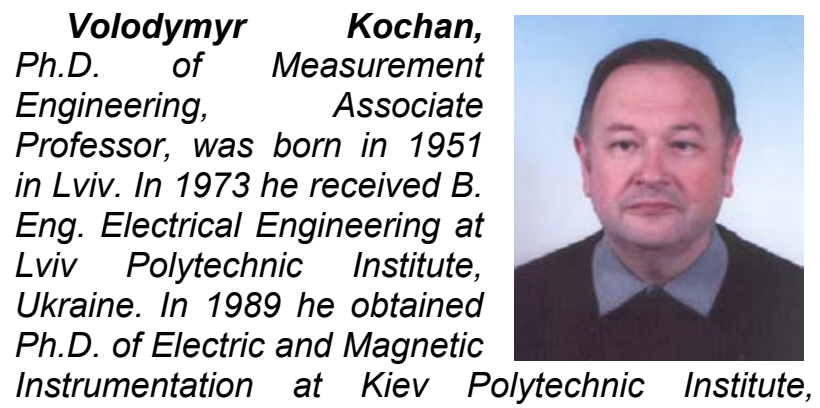
Ukraine.
Now he works as Associate Professor of Department of Specialized Computing Systems and Senior staff scientist of Research Lab. of Automation Systems and Networks of Institute of Computer Information Technologies of Ternopil Academy of National Economy; Department of Computer Science of Ternopil State Technical University; Department of Electrical devices of Technical college of Ternopil State Technical University; Instructor of the practical course on microprocessor application.

His research area includes: Sensor Intelligent System; Distributed Sensor Network; Computer based Intelligent Measurement and Control Systems; Intelligent Controllers for Automated and Robotic Systems in Industry; Sensor Systems Calibration and Verification.

\section{Anatoly Sachenko is} Professor and Director of the Institute of Computer Information Technologies, Ternopil Academy of National Economy since 1994. At present he is Visiting Fulbright Professor at University of Maine, Department of Computer Science for the 2002/03 academic year.

$\mathrm{He}$ earned his B.Eng. Degree in Electrical Engineering at L'viv Polytechnic Institute in 1968 and his PhD Degree in Electrical Engineering at L'viv Physics and Mechanics Institute in 1978 and his Doctor of Technical Sciences Degree in Electrical and Computer Engineering at Leningrad Electrotechnic Institute in1988. Since 1991 he has been Honored Inventor of Ukraine, since 1993 he has been IEEE Senior Member.

His main Areas of Research Interest are Implementation of Artificial Neural Network, , Distributed System and Network, Parallel Computing, Intelligent Controllers for Automated and Robotics Systems. He has published over 300 papers in areas above. 